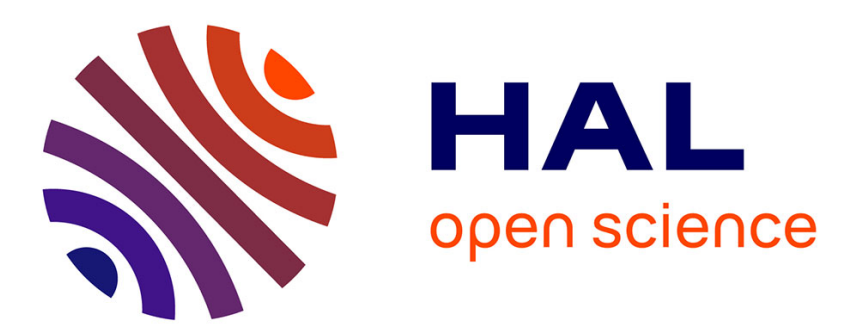

\title{
Les faux-semblants du droit de l'inventeur ou l'examen clandestin des brevets d'invention dans la France de la Belle Époque.
}

Gabriel Galvez-Behar

\section{- To cite this version: \\ Gabriel Galvez-Behar. Les faux-semblants du droit de l'inventeur ou l'examen clandestin des brevets d'invention dans la France de la Belle Époque.. Documents pour l'histoire des techniques, 2009, 17, pp.98-105. halshs-00433812}

\section{HAL Id: halshs-00433812 \\ https://shs.hal.science/halshs-00433812}

Submitted on 24 Oct 2010

HAL is a multi-disciplinary open access archive for the deposit and dissemination of scientific research documents, whether they are published or not. The documents may come from teaching and research institutions in France or abroad, or from public or private research centers.
L'archive ouverte pluridisciplinaire HAL, est destinée au dépôt et à la diffusion de documents scientifiques de niveau recherche, publiés ou non, émanant des établissements d'enseignement et de recherche français ou étrangers, des laboratoires publics ou privés. 


\section{LES FAUX-SEMBLANTS DU DROIT DE L'INVENTEUR OU L'EXAMEN CLANDESTIN DES BREVETS d'inVention dans la France de la Belle Époque ${ }^{1}$}

\section{Gabriel GALVEZ-BEHAR}

Assimilé dès 1791 à un « droit inaliénable de l'homme », le droit de l'inventeur repose sur une puissante idéologie individualiste et libérale. Le brevet d'invention, qui naît en France en 1791, atteste de la propriété de l'inventeur sur son œuvre et participe dans le même d'une individualisation du processus d'invention ${ }^{2}$. Les lois de 1791 semblent ainsi ouvrir une longue période de stabilité juridique puisque les grandes lois qui leur succèdent en la matière datent de 1844 et de 1968 . Pourtant, cette histoire immobile n'est qu'apparente : dès les premières semaines de son entrée en vigueur, et tout au long du XIX ${ }^{\mathrm{e}}$ siècle, le brevet d'invention se voit violemment contesté. Si la force de la philosophie qui l'inspire, conjuguée à une configuration historique et sociale singulière, permettent son maintien, les usages du brevet révèlent l'écart pouvant exister entre le droit proclamé de l'inventeur et le droit vécu.

L'analyse de l'examen clandestin des brevets mené par le Comité consultatif des arts et manufactures au début $\mathrm{du} \mathrm{XX}^{\mathrm{e}}$ siècle montre bien que l'on ne peut se contenter de la définition formelle d'une institution pour en apprécier les usages. Car, en apparence, la loi du 5 juillet 1844 est claire; son article 11 dispose que : « les brevets dont la demande aura été régulièrement formée seront délivrés sans examen préalable, aux risques et périls des demandeurs, et sans garantie soit de la réalité, de la nouveauté ou du mérite de l'invention, soit de la fidélité ou de l'exactitude de la demande. » À l'instar de la Belgique, de l'Italie ou de l'Espagne, la France est un pays de nonexamen préalable. Pourtant, au début du $\mathrm{XX}^{\mathrm{e}}$ siècle, soit plus de cinquante ans après l'adoption de

1. Cet article reprend les conclusions d'une communication présentée lors du colloque « Les brevets dans l'histoire » organisé en 2001 par le Centre de recherche en histoire de l'innovation de l'université Paris IV.

2. Pour une histoire plus large des usages du brevet au XIX ${ }^{\mathrm{e}}$ siècle en France, je renvoie à mon ouvrage : Gabriel Galvez-Behar, La République des inventeurs. Propriété et organisation de l'innovation en France (1791-1922), Rennes, Presses universitaires de Rennes, 2008. 
la loi de 1844 , le non-examen préalable reste l'objet de débats et de controverses. Au discours libéral qui refuse tout contrôle de l'État en la matière s'oppose un discours pragmatique qui repose sur l'apparente réussite du système d'examen allemand, considéré comme l'un des facteurs du développement industriel de l'Allemagne ${ }^{1}$. En outre, si elles ne connaissent pas de succès, les tentatives d'introduction d'un examen préalable dans la législation française illustrent la vitalité d'un débat récurrent au sein des milieux intéressés par les problèmes de propriété industrielle².

En fait, le débat se pérennise du fait des imperfections d'une législation moins claire qu'il n'y paraît. Si la loi de 1844 rend impossible la brevetabilité des compositions pharmaceutiques, des remèdes pharmaceutiques ou combinaisons financières, l'Administration doit s'assurer elle-même du respect de ces critères. Cela rend « son investigation nécessaire alors même que, pour respecter le principe du non-examen préalable, elle [doit] limiter son examen au seul titre de l'invention sans pouvoir même en consulter la description. $»^{3}$ C'est là l'une des contradictions de la loi qui introduit un contrôle administratif là où le non-examen est proclamé. Au début du $\mathrm{XX}^{\mathrm{e}}$ siècle, ce contrôle est assuré par l'Office national de la propriété industrielle - et plus particulièrement par son comité technique - et par le Comité consultatif des arts et manufactures ${ }^{4}$. C'est ce contrôle préliminaire effectué par le Comité consultatif des arts et manufactures que nous souhaitons examiner de plus près afin d'illustrer cette notion paradoxale de non-examen préalable et de montrer, par ailleurs, la complexité du brevet d'invention et de la propriété industrielle 5 .

1. L'Empire allemand se dote d'une nouvelle législation sur le « droit de l'invention » en 1877 et 1891 . Gabriel GalvezBehar, «L'État et l'invention : une comparaison franco-allemande des cadres de la propriété industrielle à la fin du XIXe siècle » in Alain Chatriot et Dieter Gosewinkel (sous la dir.), Figurationen des Staates, 1870-1945. Les figures de l'État, Munich, Oldenbourg, 2006, p. 215-234.

2. Plusieurs tentatives de réformes sont faites dans la première décennie du $\mathrm{XX}^{\mathrm{e}}$ siècle : par le député Allemane en 1907, par le député Astier, président de la Commission du commerce et de l'industrie à la Chambre en 1908 et par le ministre Cruppi en 1909.

3. Jules Delagrange, De l'examen préalable en matière de brevets d'invention, thèse pour le doctorat de droit, Paris, M. Giard et É. Brière, 1912, p. 28. Il ajoute « sauf cependant si, au cours de l'examen de pure forme, il apparaît que le titre ne révèle pas exactement la vraie nature de l'invention ».

4. L'ONPI est créé en 1901 dans le cadre d'une réforme du Conservatoire national des arts et métiers. Cette création a pour but d'initier la centralisation des organismes officiels concernés par la propriété industrielle.

5. Cette étude repose sur l'analyse des rapports dont nous disposons sur la période courant de 1903 à 1914. 


\section{Le Comité consultatif des arts et manufactures et les brevets}

Le Comité consultatif des arts et des manufactures remonte à la création, en 1791, du Bureau de consultation des arts et métiers, chargé alors de répartir une subvention globale assignée aux inventeurs indigents, et à la création en l'an II d'une Agence des arts et manufactures destinée à promouvoir l'industrie et composée, notamment, de fonctionnaires comme Louis Costaz et ClaudePierre Molard ${ }^{1}$. Parce que «l'administration [...] rencontre une foule de questions techniques dont la solution exige le concours d'hommes versés, les uns dans les sciences exactes, et les autres dans l'économie manufacturière » cet organisme se maintient sous le Directoire sous le nom de Bureau consultatif des arts et des manufactures, ses missions perdurant ensuite tout au long du XIX siècle ${ }^{2}$. Comme le répètent les dispositions réglementaires, il reste «chargé de l'étude et de l'examen de toutes les questions intéressant le commerce et l'industrie qui lui sont renvoyées par le ministre en vertu des lois et règlements, ou sur lesquelles le ministre juge utile de le consulter, notamment en ce qui concerne: les établissements insalubres ou incommodes; les brevets d'invention ; l'application ou la modification, au point de vue technique, des tarifs et des lois de douanes. Il peut être chargé de procéder aux enquêtes ou informations qui sont jugées nécessaires par le ministre pour l'étude des questions ci-dessus énoncées. $»^{3}$

Si ces textes réglementaires amènent périodiquement une redéfinition du nombre de ses membres (15 en 1869, 20 depuis 1880), ils ne remettent pas en question le caractère pluraliste du comité qui rassemble des membres de la haute administration, des corps techniques de l'État et des institutions savantes. Ainsi, en 1900, le Comité est présidé par Eleuthère Mascart, membre de

1. Dominique De Place, «Le Bureau de Consultation pour les Arts, 1791-1796 », History and Technology, vol. 5, 1988, p. 139-178. Serge Chassagne, «L'enquête dite de Champagny, sur la situation de l'industrie cotonnière française au début de l'Empire », Revue d'histoire économique et sociale, vol. 54, 1976, p. 336-370, note 8.

2. Maurice Block, Dictionnaire de l'administration française, Paris, Berger-Levrault, 1877. Sur les débuts du Comité consultatif des arts et manufactures, voir Igor Moullier, Le ministère de l'Intérieur sous le Consulat et le Premier Empire (1799-1814), thèse de doctorat sous la dir. de G. Gayot, Université Lille 3, 2004, p. 211-214.

3. « Décret du 18 octobre 1880 portant réorganisation du Comité consultatif des arts et manufactures » in Bulletin des lois de la République française, $12^{\mathrm{eme}}$ série, tome 21, Deuxième semestre de l'année 1880, Paris, Imprimerie nationale, 1881, p. 1095, art. 1. 
l'Académie des sciences, physicien, et professeur au Collège de France. Sur les 24 membres effectifs, on compte trois membres de l'académie des Sciences, cinq directeurs du ministère du Commerce et de l'Industrie (membres de droit), le directeur du Conservatoire national des arts et métiers, deux présidents de section du Conseil d'État, trois membres des corps des Mines et des Ponts et Chaussées et, enfin, cinq représentants du commerce ou de l'industrie ${ }^{1}$. Cette commission n'est pas une commission fantôme : elle se réunit une fois par semaine et ses membres ne sont pas absentéistes. Entre 1904 et 1907, en moyenne, les trois quarts des membres participent directement aux séances $^{2}$. Il est vrai que le versement de jetons de présence (15 francs par séance ou 1500 francs si l'on a assisté à une cinquantaine de séances au moins) est une incitation non négligeable. Mais ces derniers rétribuent un travail réel qui nécessite parfois des déplacements et des expertises sur le terrain. Instance d'experts de haut niveau, le Comité consultatif des arts et manufactures se voit donc confier des questions à la fois juridiques, économiques, techniques et scientifiques. Les problèmes liés aux brevets d'invention font partie, on l'a vu, de son champ de compétence.

Dès le tout début du XIX ${ }^{\mathrm{e}}$ siècle, le Bureau consultatif intervient en effet dans la délivrance des brevets. En 1811, le Conseil général des fabriques et des manufactures reconnaît en effet que le Bureau procède à un examen préalable afin d'aider les inventeurs à améliorer la rédaction de leurs demandes et à les informer de l'existence d'éventuelles antériorités ${ }^{3}$. Plus tard, dans les années 1820, le Comité consultatif traite de 300 à 600 brevets par an et près de 1300 à 1600 à la fin de la décennie suivante ${ }^{4}$ L'intervention du Comité dans ce domaine est donc un usage bien ancré. Il semble bien, cependant, que la loi de 1844 ait quelque peu restreint cette pratique. Non seulement elle réaffirme le principe du non-examen préalable, mais encore les débats qui précèdent son

1. Almanach National, annuaire officiel de la République française pour l'année 1900, Paris, Berger-Levrault et Cie, 1901.

2. Calcul réalisé à partir des rapports concernant les brevets d'invention. Archives nationales (désormais AN), $\mathrm{F}^{12} 7496$. 3. AN, $\mathrm{F}^{12} 194$, séance du 27 mai 1811.

4. Jean-Pierre Hirsch et Philippe Minard, " "Laissez-nous faire et protégez-nous beaucoup" : pour une histoire des pratiques institutionnelles dans l'industrie française (XVII ${ }^{\mathrm{eme}}-\mathrm{XVIII}{ }^{\mathrm{eme}}$ siècle) » in Louis Bergeron et Patrice Bourdelais (sous la dir. de), La France n'est-elle pas douée pour l'industrie, Paris, Belin, 1998, p. 135-158. 
adoption conduisent à limiter le regard du Comité : ce dernier n'ayant pas à juger des brevets au fond, il doit se contenter de procéder à une lecture du titre de la description pour juger de la régularité de la demande. Comme le dit Ambroise Rendu, avocat spécialiste de propriété industrielle dans les années 1870, «c'est seulement d'après l'intitulé de la demande [...] que le rejet devra être prononcé $\gg{ }^{1}$.

La création de l'ONPI en 1901 et l'adoption de nouvelles règles relatives à la forme des demandes viennent toutefois accentuer le rôle de l'administration dans la délivrance des brevets. Ainsi la loi du 7 avril 1902 confère-t-elle au ministre du Commerce et de l'Industrie le pouvoir de préciser les conditions de forme et de rédaction des descriptions, tandis que l'arrêté du 11 août 1903 renforce le droit de regard du Comité technique de l'ONPI sur les demandes. En 1908, le service technique de l'Office, composé de trois ingénieurs des arts et manufactures, étudie environ 15000 brevets par an, «dont 3000, au moins, donnent lieu à des corrections qui nécessitent un second examen $»^{2}$. L'Office procède donc à un premier tri, ce qui explique la faiblesse du nombre de cas examinés par le Comité consultatif. En effet, les rapports dont nous disposons pour étayer notre analyse sont seulement au nombre de 87 pour la période allant de 1903 à 1914. Si une part de ce chiffre peut éventuellement s'expliquer par des lacunes dans les sources, il n'en demeure pas moins que, contrairement au début du $\mathrm{XIX}^{\mathrm{e}}$ siècle, le Comité consultatif semble bien n'être saisi que d'un petit nombre d'affaires, mais sans doute parmi les plus sensibles ${ }^{3}$. C'est en effet le directeur de l'ONPI qui sollicite l'avis du Comité consultatif avant de proposer une décision de rejet au ministre.

Si le contrôle des brevets ne constitue pas l'essentiel de l'activité du Comité, certains de ses

1. Ambroise Rendu, Codes de la propriété industrielle, tome 1, Brevets d'invention, Paris, Durand et Pédone, 1879 , p. 82. Ce principe est réaffirmé par de nombreux auteurs et notamment Michel Pelletier, Droit industriel. Brevets d'invention, marques de fabrique, modèles et dessins, nom commercial, concurrence déloyale, Paris, Baudry, 1893, p. 36 ainsi que Jules Delagrange, loc. cit.

2. Claude Couhin, Rapport sur le fonctionnement de l'Office national de la propriété industrielle, Paris, 1908.

3. Entre octobre 1912 et avril 1913, sur 78 affaires renvoyées à des rapporteurs, 13 concernaient les demandes de brevet ; par extrapolation on peut donc établir cette moyenne de 24 affaires annuelles. Or nous ne disposons que de huit rapports en moyenne par an. $\mathrm{AN}, \mathrm{F}^{22} 312$ 
membres se spécialisent dans cette tâche : trois rapporteurs réalisent ainsi un peu moins de $60 \%$ des rapports et le secrétaire du Comité se voit confier plus de trois rapports sur dix ${ }^{1}$. Le principal motif de saisine concerne les remèdes et compositions pharmaceutiques ( $72 \%$ des rapports environ) et les rapports sont avant tout confiés au secrétaire et aux chimistes du Comité : Haller, Müntz et dans une moindre mesure Schlœsing. Sans doute ces derniers sont-ils considérés comme étant mieux à même de déterminer si l'objet de la demande de brevet correspond ou non à un remède ou à une composition pharmaceutique ${ }^{2}$. L'activité du Comité consultatif ne se limite cependant pas aux seules affaires relatives aux remèdes et aux compositions pharmaceutiques. Près de $17 \%$ des cas se rapportent ainsi à des problèmes de complexité des demandes et sont, là aussi, traités par des spécialistes : Liébaut, constructeur mécanicien, Lodin, professeur à l'École des Mines ou Moron, ingénieur en chef des Ponts et Chaussées ${ }^{3}$. Les quelques cas posés par des demandes semblant porter atteinte aux mœurs ou à l'ordre public sont essentiellement traités par le secrétaire du Comité. Le Comité consultatif des arts et manufactures apparaît donc la haute instance d'expertise permettant de compléter le contrôle préliminaire à la délivrance des brevets d'invention.

Il n'est guère aisé d'analyser l'identité des demandeurs des brevets examinés. Les rapports, le plus souvent, ne permettent d'en connaître que le nom. Seuls vingt-quatre d'entre eux sont clairement identifiés comme étant de nationalité étrangère et la moitié seulement voient leurs demandes acceptées. Faut-il voir là la marque d'une méconnaissance plus grande de la législation française en matière de propriété industrielle ou les traces d'un protectionnisme larvé ? Trop peu d'éléments statistiques permettent de discuter une telle hypothèse. Mais dans $57 \%$ de l'ensemble des cas, le rapporteur chargé de l'examen de la demande suggère au Comité consultatif son

1. Ces rapporteurs sont E.P. Bérard (30 \%), A. Haller (15\%) et A. Müntz (14 \%).

2. Sur la brevetabilité du médicament, voir Maurice Cassier, «Brevets pharmaceutiques et santé publique en France : opposition et dispositifs spécifiques d'appropriation des médicaments en France entre 1791 et 2004 », Entreprises et histoire, octobre 2004, n 36, p. 29-47 ; id., «Patents and public health in France. Pharmaceutical patent law in-themaking at the patent office between the two world wars », History and Technlogy, juin 2008, vol. 24, n ${ }^{\circ}$ 2, p. 135-151.

3. Un brevet d'invention doit avoir un seul objet; un brevet visant à breveter plusieurs procédés est entaché de complexité. 
acceptation : les décisions sont donc assez équilibrées d'autant que l'avis du Comité est quasiment toujours suivi. Autant que l'on peut en juger, il semble bien que les services techniques de l'ONPI ne transmettent pas au Comité consultatif des arts et manufactures des cas jugés d'avance. Les rapporteurs fournissent un réel travail et savent d'ailleurs prendre leur temps puisqu'en moyenne, les avis sont donnés un mois et demi après la saisine du Comité par le ministre.

L'étude globale des rapports du Comité permet donc d'apprécier la place prise par l'examen des brevets dans l'activité de cette haute instance d'expertise qui se voit soumettre des cas bien particuliers soit par leur nature soit par leur complexité. Elle ne nous dit rien, en revanche, du regard que portent les différents rapporteurs sur la nature même du brevet d'invention. Pour cela il faut se pencher sur les pratiques.

\section{$\underline{\text { L'herméneutique du brevet }}$}

L'élaboration des rapports du Comité est fortement contrainte par la règle du non-examen préalable. Les rapporteurs du Comité sont tout à fait conscients que leur contrôle ne doit pas paraître constituer un examen préalable en totale contradiction avec la législation et susceptible d'un recours devant le Conseil d'État. Pourtant les membres du Comité consultatif n'hésitent pas à aller au-delà des limites encore considérées comme légitimes à la toute fin du XIX ${ }^{\mathrm{e}}$ siècle. Loin de se limiter à la lecture des intitulés, ils prétendent fonder leur examen sur le texte de la description et, éventuellement, des schémas joints. Mais pour éviter toute contestation, les voilà obligés d'insister dans leurs rapports sur le caractère superficiel de leur lecture en y multipliant les citations et l'emploi de termes mettant en avant leur statut de simple lecteur. C'est ce qu'illustre le rapport consacré à l'étude d'« un procédé servant à obtenir un dérivé de l'acide cantharidique contenant de l'iode et du mercure » :

«"Lorsque l'on mélange, dit le pétitionnaire, de la teinture de cantharides ou une solution d'acide cantharidique avec une solution de chlorure mercurique, on obtient un précipité blanc que l'on avait déjà obtenu précédemment et qui, vu son action caustique sur les exastases des chevaux et surtout sur 
les hypertrophies et néoformations osseuses, rend de grands services" [...] et plus loin on lit : " ce produit peut être employé ou bien dans l'alcool ou dans d'autres produits médicamenteux" »

De la sorte, le rapporteur suggère bel et bien les conditions de son propre effacement et tente de faire apparaître la recevabilité ou l'irrecevabilité de la demande en se référant uniquement à une lecture au premier degré et à des citations bien choisies ${ }^{1}$. La place du jugement ou de l'interprétation doit être réduite au minimum dans l'écriture des rapports alors qu'elle est prédominante dans l'analyse des brevets. C'est ce qu'exprime, de manière assez péremptoire, le secrétaire du Comité dans son rapport sur la demande de brevet pour une lotion contre la chute des cheveux en 1904 : «Le Comité ne juge pas les brevets au fond : il appuie ses avis sur les dires des auteurs. $»^{2}$

En fait, ce mot d'ordre censé rappeler l'attachement du Comité au principe de non-examen est largement illusoire. Le secrétaire du Comité en donne d'ailleurs lui-même la preuve. Loin de s'en tenir aux seules affirmations de l'auteur de la demande qui présente l'invention comme une lotion contre la chute des cheveux - titre qui pourrait suffire à la qualifier de cosmétique et non de remède -, Bérard, secrétaire du Comité, analyse la composition du produit qui contient, outre de l'infusion de fleur de camomille, de la dissolution d'air marin et de petites quantités d'iode, une poudre antiseptique de « Fluoral ». Et le rapporteur d'affirmer : « nous ne savons pas qu'elle est la matière que l'auteur désigne sous ce nom : plusieurs personnes très compétentes que nous avons consultées n'ont pas pu nous renseigner à son sujet $»^{3}$. Ainsi, au moment même où il affirme que le Comité ne doit pas procéder à un examen fondamental du brevet, Bérard avoue avoir effectué une enquête sur le fond, recourant même aux témoignages de spécialistes. Certes, le secrétaire du Comité ne s'exprime pas sur la nouveauté, l'utilité ou l'efficacité du brevet mais il montre bien que la seule lecture des descriptions - et encore moins celle du titre - ne suffit pas à asseoir son jugement.

1. AN, $\mathrm{F}^{12} 7496$, séance du 4 décembre 1907.

2. AN, $F^{12} 7496$, séance du 8 novembre 1904.

3. Idem 
L'enquête du Comité consultatif se fonde parfois sur une véritable tentative d'interprétation des demandes. En effet, les rapporteurs sont parfois confrontés à des descriptions dont ils ont du mal à cerner le sens. C'est ce qu'illustre l'extrait d'un rapport portant sur une demande pour un procédé de fabrication à partir de kéfiri ${ }^{1}$ :

\begin{abstract}
«Et quant à l'usage auquel l'auteur destine ce produit, il est indiqué par le passage suivant que nous relevons dans la demande : "[Le produit] donne du sang et quand il est traité de manière spéciale il remplit les tubercules de sels calcaires chez les tuberculeux" et plus bas : "le goût du produit ne change pas, ce qui est très important pour les tuberculeux, ceux-ci pouvant le boire sans avoir la mention (sic) d'absorber un médicament spécialement nécessaire à leur guérison". Les citations prouvent, à notre sens, que le kéfir, serait, dans la pensée de l'auteur, le véhicule d'un médicament dont, bien entendu, nous lui laissons la responsabilité et qui serait l'objet de la demande $»^{2}$.
\end{abstract}

Face à une description qui lui paraît obscure, le rapporteur ne s'en tient plus seulement au texte de la description mais tente de s'appuyer sur l'intention présumée de l'auteur. Cette méthode, largement interprétative, montre bien qu'une lecture simple et neutre du brevet n'est pas toujours possible. Elle sera d'ailleurs l'objet, on le verra, de contestations au sein même de l'Administration.

Le contrôle préliminaire effectué par le Comité ne repose donc pas sur une simple lecture des descriptions. La place de l'interprétation y est d'autant plus fondamentale que, parfois, les membres du Comité consultatif doivent faire face à des tentatives de contournement de la part des requérants. Parce qu'elle repose essentiellement sur un texte, la demande de brevet fait l'objet de véritables stratégies d'écriture. Ainsi, quand il dépose sa demande pour la «préparation de limonades purgatives », l'Institut expérimental universel prend garde de ne pas demander son brevet pour le produit lui-même mais pour le procédé de préparation. Le pétitionnaire se contente alors de décrire le mélange des produits dans sa demande. Point dupe, le rapporteur propose tout de même le rejet de la demande invoquant la jurisprudence du Comité en la matière qui consiste à refuser tout brevet relatif à un procédé qui entraînerait le monopole du produit pharmaceutique ${ }^{3}$. On mesure là combien la décision du rapporteur repose sur une véritable évaluation de la demande qui contredit le principe

1. AN, $\mathrm{F}^{12} 7496$, séance du 18 octobre 1905.

2. Idem. Souligné par nous.

3. AN, $\mathrm{F}^{12} 7496$, séance du 18 décembre 1907. 
légal de non-examen.

Cette herméneutique du brevet est d'ailleurs d'autant plus délicate que le Comité est confronté à des inventions touchant aux mœurs et à la sexualité. L'attitude du Comité face à certains appareils gynécologiques et aux instruments préservatifs est alors très soupçonneuse. Il n'est pas question, en effet, de déclarer recevables des demandes portant sur des inventions susceptibles de servir de contraceptifs. Le Comité doit alors fonder un principe d'interprétation qui lui permet de rejeter de tels brevets sans pour autant quitter la posture de lecteur au premier degré des descriptions. Ainsi, en 1902, lorsqu'il étudie la demande faite pour un dispositif de fixation pour pessaires, le Comité s'appuie - s'accroche même - à des affirmations de l'inventeur pour motiver son refus ${ }^{1}$. Le fait que l'inventeur affirme que «[son] invention se reporte à un mode de fermeture rationnel de la partie supérieure des organes sexuels de la femme » ou bien encore que « la disposition du pessaire qui forme l'objet de l'invention assure une bonne fermeture de la matrice » suffit au Comité pour voir «dans ces indications répétées une visée immorale $»^{2}$. En revanche, cinq ans plus tard, le directeur de l'ONPI s'étonne auprès du ministre du Commerce et de l'Industrie de ce que le Comité ne s'oppose pas à la délivrance d'un brevet alors que son rapporteur reconnaît que l'appareil, « une houppette en fil de soie soutenue sur une tige », " étant donné les formes, dispositions et mode d'introduction, [peut] servir aux pratiques de galanterie ou comme préservatif. $»^{3}$ À nouveau saisi par le ministre, le Comité se défend en ces termes :

«Un semblable pansement est le but que vise, du moins d'après les affirmations de l'auteur de la demande, la houppette qu'il décrit. M. le Directeur admet que cette houppette doit servir à un usage autre que l'usage médical. Mais il ne donne pas de démonstration et, en effet, on ne peut la tirer du texte. L'opinion de M. le Directeur ne peut être fondée que sur un simple soupçon, soupçon qui pourrait être étendu à bien des appareils et pratiques de l'art médical et qu'on ne saurait, sans s'écarter des textes, faire intervenir dans un avis. Serait-il permis de rejeter les demandes pour appareils destinés aux douches vaginales, lesquelles sont d'un usage courant dans le traitement des maladies des femmes pour le motif que certaines femmes peuvent se servir de ces douches pour s'affranchir des

1. Le pessaire est un diaphragme permettant le maintien de l'utérus dans sa situation naturelle, en cas de chute ou de déplacement de cet organe.

2. AN, $\mathrm{F}^{12} 7496$, séance du 28 décembre 1907.

3. Ibidem, lettre du 5 novembre 1917 du directeur de l'ONPI au ministre du Commerce et de l'Industrie. 
suites naturelles des approches de $l^{\prime}$ homme ? ${ }^{1}$

Le Comité accepte donc de lutter contre les techniques portant atteinte aux bonnes mœurs, en s'en tenant toutefois au texte du brevet.

\section{Des pratiques contestées}

Pourtant, de tels scrupules peuvent apparaître surprenants. Le Comité ne veut pas aller au-delà des textes mais il accepte d'enfreindre la loi de 1844 n'offre pas à l'Administration de moyens réels pour refuser ce type de brevets. En effet, si elle considère comme nuls les brevets d'invention portant atteinte aux bonnes mœurs ou à l'ordre public, elle ne permet pas à l'Administration de les refuser s'ils sont demandés dans les règles : seul un tribunal, saisi par le ministère public, peut déclarer nul un brevet délivré. En outre, le Comité n'hésite pas à reconnaître, en effet, le caractère relatif de ses décisions. Ainsi, au directeur de l'ONPI qui lui reproche d'avoir pris deux décisions contradictoires pour deux objets similaires, le Comité répond : «La matière des brevets, par sa diversité, par le caractère obscur des idées émises, par l'incorrection ou l'insuffisance volontaire ou voulue des descriptions, se prête mal à la Jurisprudence fixe qu'invoque M. le Directeur. Les questions qui se posent à son sujet ne peuvent être résolues que comme des cas d'espèce. $»^{2}$

Pourtant, en 1903, le Comité tente de donner un fondement juridique à ses propres infractions. Un rapport du président de la section du contentieux au Conseil d'État, Berger, fixe en effet la ligne du Comité en affirmant que l'Administration ne peut délivrer un brevet dont l'objet serait contraire à l'ordre public ou aux bonnes mœurs. Dix ans plus tard, le refus opposé au brevet de M. Bihari pour des «perfectionnements apportés aux protecteurs hygiéniques contre les maladies véneriennes » provoque un contentieux qui aboutit à un arrêt du conseil d'État ${ }^{3}$. Le Comité consultatif doit alors abandonner sa « jurisprudence » mais propose au directeur de l'ONPI ne faire saisir au plus vite le ministère public afin que celui-ci prenne les mesures nécessaires pour faire

1. Ibidem. Souligné par nous.

2. AN, $\mathrm{F}^{12}$ 7496, séance du 28 décembre 1907.

3. Arrêt du Conseil d'État du 7 février 1913 mentionné en AN F ${ }^{12} 7602$. 
procéder à l'annulation du brevet ${ }^{1}$. À défaut de pouvoir empêcher la délivrance du brevet, le Comité entend toujours empêcher la protection d'une technique demeurant morale aux yeux de ses membres, masculins dans leur ensemble.

L'attitude du Comité illustre donc le caractère illusoire, pour un nombre de cas limité il est vrai, du principe de non-examen préalable. En ce qui concerne les problèmes liés aux instruments gynécologiques ou préservatifs, les membres du Comité n'hésitent pas à prononcer, de manière implicite ou non, un jugement quant à la moralité ou l'utilité sociale de la demande examinée, quitte à dépasser le strict cadre de leurs prérogatives. Ce contrôle préliminaire est donc l'occasion de défendre à la fois le respect des dispositions législatives, celui d'un certain code moral mais aussi les intérêts supposés de l'Administration et de l'État. Donnant le sentiment de frôler parfois l'arbitraire, l'attitude du Comité donne lieu à des contestations de la part de l'Administration ellemême.

Malgré l'importance des personnages qui les rendent, les décisions du Comité consultatif ne sont pas acceptées sans qu'un contrôle ne soit effectué en aval. Le directeur de l'ONPI est, en effet, très attentif aux décisions rendues. À plusieurs reprises, il les conteste en soulignant les contradictions du Comité dans le temps ou en remettant en cause le contenu même des rapports. Ainsi, par une lettre du 17 décembre 1910 au ministre du Commerce et de l'Industrie, le directeur de l'ONPI critique le rapport rendu sur une demande pour des bandes de pansement pour emplâtre. C'est surtout la forme même du rapport que le directeur remet ici en cause. Il souligne l'inexactitude des faits avancés par le rapporteur et lui reproche l'emploi de certaines formulations laissant penser que le Comité ne s'est pas contenté de l'examen des seuls éléments intrinsèques qui constituent l'affaire ${ }^{2}$. Et d'ajouter, cassant : «Il semble s'être produit, tout au moins, une confusion 1. $\mathrm{AN} \mathrm{F}^{12} 7602$

2. «Si l'on devait rejeter la présente demande par le motif que “dans l'esprit de l'inventeur" la bande de pansement doit ajouter aux propriétés curatives de l'emplâtre auquel elle sert de support, ne serait-il pas à craindre que cet argument ne parût avoir un caractère tendancieux et qu'il eût par la suite peu de poids dans le cas d'un pourvoi qu'il faut toujours envisager comme possible en la matière ». AN, $\mathrm{F}^{12} 7496$, séance du 12 octobre 1910. 
qui, sans rien changer vraisemblablement à l'avis du Comité, s'est traduite, en la forme, par une rédaction qui ne répond pas exactement à la réalité des faits. Mais quand il s'agit de la préparation d'une décision susceptible de faire l'objet d'un recours au Conseil d'État, les questions de forme ne sont pas négligeables » ${ }^{1}$. Chargé de «sauvegarder la responsabilité de l'Administration », le directeur de l'ONPI n'hésite pas à s'opposer aux menées risquées du Comité.

Individuellement responsable de son action auprès du Ministre, le directeur de l'ONPI l'est aussi, dans une certaine mesure, auprès des inventeurs et des agents de brevets ${ }^{2}$. La pression des inventeurs n'est sans doute pas la plus forte, bien qu'elle puisse être, parfois, réelle et pressante. Ainsi le directeur de l'Office signale-t-il en 1904 au ministre du Commerce et de l'Industrie le cas d'un inventeur furieux d'avoir vu sa demande de certificat d'addition rejetée et forçant l'entrée de son bureau « le gourdin à la main et la menace à la bouche ». Stoïque, le directeur déclare : «Ce n'est pas là un fait dont il y a lieu d'être surpris, car au nombre de 12 ou 13000 inventeurs qui prennent des brevets chaque année, il y en a nécessairement qui, ne jouissant pas de toutes leurs facultés, se montrent plus ou moins violents. C'est là une situation dont le Directeur de l'Office a depuis longtemps pris son parti. » Mais, soucieux de la sécurité de ses collègues, il ajoute : « Mais M. Dugenait [a] manifesté l'intention d'aller au Ministère où il pourrait aller provoquer un scandale $»^{3}$.

La relation que le directeur de l'Office entretient avec les agents de brevets, dont le rôle est essentiel dans la procédure de demande, est plus apaisée. Ainsi, 62\% des inventeurs dont les demandes ont été soumises au Comité consultatif sont-ils représentés par un mandataire ou par un agent comme Blétry, Armengaud jeune ou Chassevent ${ }^{4}$. Le rôle de ces derniers ne s'arrête d'ailleurs

\section{Idem}

2. Sur les agents de brevets, voir Gabriel Galvez-Behar, « Des médiateurs au cœur du système d'innovation. Les agents de brevets en France, 1870-1914 », in Marie-Sophie Corcy, Christiane Douyère-Demeulenaere et Liliane Hilaire-Pérez (sous la dir. de), Les archives de l'invention. Écrits, objets et images de l'activité inventive, Toulouse, CNRS-Université Toulouse-Le Mirail, 2006, p.437-447.

3. AN, $F^{12} 7496$, séance du $27 / 07 / 1904$.

4. Tous les mandataires ne sont pas, effectivement, agents de brevet. Si le mandataire dépose le brevet au nom de 
pas au guichet de l'ONPI; les agents n'hésitent pas à entrer directement en relation avec le directeur de l'Office lorsqu'un problème se présente et sont tout à fait prêts à opposer les recours appropriés lorsqu'ils jugent qu'une décision lèse les intérêts de leurs mandants. C'est ce qu'atteste un échange entre le directeur de l'Office et le cabinet d'Armengaud jeune en 1907. L'examen de la demande effectuée par Armengaud jeune est l'occasion d'un véritable dialogue entre ce dernier et l'ONPI. En janvier 1907, le service technique produit un rapport soulignant la complexité de l'appareil inventé. L'ONPI adresse alors deux courriers au cabinet Armengaud jeune pour lui signaler le rejet probable de la demande. Sans réponse, le directeur de l'Office renvoie donc l'affaire devant le Comité qui rejette la demande en avril. Le $1^{\text {er }}$ juin, l'ONPI informe Armengaud jeune qui répond que l'inventeur est prêt à modifier son projet et désire être mis en présence du rapporteur pour lui démontrer l'unité de sa demande. Le directeur de l'ONPI prend alors acte de cette réponse et annonce que le Comité consulatif sera de nouveau consulté.

Ce dernier refuse pourtant catégoriquement de recevoir l'inventeur car « c'est une voie dans laquelle le Comité ne doit pas entrer », au nom du principe de non-examen préalable, et maintient son avis. ${ }^{1}$. Sa marge de manœuvre paraît ainsi d'autant plus grande qu'il n'est pas directement confronté aux réclamations des inventeurs et de leurs agents. Pourtant, vers les années 1910, une évolution se dessine : les agents de brevets n'hésitent plus alors à faire figurer dans le dossier soumis au Comité une lettre exposant les raisons qui militent en faveur de la brevetabilité de l'invention. La distance entre les inventeurs et cet aréopage de sommités juridiques, scientifiques et administratives tend à diminuer et si le poids des décisions de cette instance reste reconnu, ces dernières ne revêtent plus le même caractère imposant et magistral qu'elles pouvaient avoir quelques années auparavant.

quelqu'un, l'agent, lui, offre plus de services grâce à sa connaissance de la propriété industrielle. 1. AN, F $\mathrm{F}^{12} 7496$, séance du 17 avril 1897. 


\section{Conclusion}

Le traitement des brevets d'invention par le Comité consultatif des arts et manufactures suggère quelques conclusions. En premier lieu, il faut souligner l'importance de l'aspect textuel du brevet. Comme on l'a vu, la lecture du brevet n'est pas toujours simple et passe le plus souvent par une interprétation requérant des compétences d'analyse complexes. De fait, le brevet est un objet dont la construction ne va pas de soi et qui peut donner lieu à l'emploi de véritables stratégies d'écriture permettant de répondre aux contraintes contradictoires du brevet. Les agents de brevet sont d'ailleurs ceux qui sont au fait de ces stratégies et qui connaissent les règles de l'art. On pourra certes objecter qu'une telle conclusion repose sur l'analyse de cas limites. Outre que l'on peut renvoyer aux mérites de «l'exceptionnel normal», on peut aussi noter que la place de l'interprétation du brevet est précisément accentuée par le principe de non-examen préalable proclamée par la loi de 1844. En effet, cette dernière fait des tribunaux une véritable instance d'examen du brevet d'invention. Le rédacteur du brevet doit donc, pour bien faire, anticiper sur les lectures et les interprétations des juges et des experts. L'exemple du Comité consultatif ne fait sans doute que refléter, de manière certes biaisée, des pratiques employées dans les prétoires.

Le deuxième point découle du précédent. Si le brevet nécessite des compétences spécifiques, l'égalité supposée face au brevet d'invention est par conséquent une égalité formelle. L'obstacle de la taxe à payer se double alors d'un écueil plus subtil puisque ceux qui n'ont pas forcément connaissance de la jurisprudence en matière de brevet courent le risque d'être mal protégés. À la catégorie volontiers globalisante d'« inventeur» se substitue alors une pluralité d'acteurs dont l'accès à la propriété industrielle est différencié. À cette pluralité des inventeurs correspond d'ailleurs une pluralité d'attitudes au niveau de l'État. L'exemple du Comité consultatif illustre combien les intérêts poursuivis et les valeurs défendues par différents rouages d'une même administration peuvent diverger. En somme, le droit de l'inventeur ne résume en rien en un face-à- 
face entre l'individu et l'État..

Au final, le brevet d'invention ne peut pas être simplement perçu comme un simple outil juridique qui viendrait favoriser l'invention. S'il est vrai que le brevet suppose certaines règles, ces dernières ne sont pas d'une application évidente et peuvent même se révéler contradictoires. Dès lors, pour évaluer son apport aux dynamiques de l'innovation, il convient d'avoir présent à l'esprit que le brevet est aussi le lieu où se confrontent des pratiques et où convergent des acteurs. Il constitue de ce fait un objet historique à part entière.

Gabriel GALVEZ-BEHAR

Université Lille Nord de France, France

Université Lille 3, CNRS, IRHiS (Institut de Recherches Historiques du Septentrion), UMR 8529, F-59653 VILLENEUVE D'ASCQ, France 